\title{
Defects of ocular movement and fusion after head injury
}

\author{
A. STANWORTH
}

Hallamshire Hospital, Sheffield

Ocular movement defects after head injury are, of course, common and may well be of increasing frequency. They are usually discussed primarily in terms of IIIrd, IVth, and VIth nerve weakness, or pareses of individual muscles or combinations of muscles, and the management of such defects has already been discussed to a considerable degree in this symposium. In any case, the impression one has from this approach-namely that a lesion occurs at a specific point to produce a specific effect-is not necessarily correct or helpful. On the one hand, head injuries sufficient to produce concussion and ocular palsies are likely to cause widespread abnormalities of local blood flow, haemorrhage, rupture of white fibres, and cell destruction; and on the other hand, oculo-motor control is likely to be much more diffusely distributed through the midbrain than has previously been thought. Nashold and Seaber (1972), studying stereotactic lesions in the midbrain in man, came to the conclusions, for example, that convergence is not focally localized to the midline, but is more diffuse over the pretectal region, the midline structures not being the primary centre; that there is a diffuse association of accommodation and pupillary reaction which can be symmetrically affected by a unilateral lesion; that vertical gaze is also diffusely organized in the dorso-lateral midbrain; and that a unilateral lesion can cause bilateral paralysis of vertical gaze as well as a skew deviation. In view of this diffuse neural organization and the widespread effects of head injury, one wonders if it is always necessary to search for a particular point of injury to explain a particular effect, such as, for example, explaining the not uncommon bilateral superior oblique palsy by a lesion at the point where the trochlear fibres cross in the anterior medullary velum (Chapman, Urist, Folk, and Miller, 1970).

Nashold and Seaber (1972) found, incidentally, that divergence palsy was rare in their series of stereotactic lesions, as it is in head injuries, and they thought that this was because it was probably represented in a more restricted area and therefore less liable to be involved; they concluded that divergence was an active function because divergence palsy could coexist with convergence palsy.

One basic function that is likely to be diffusely represented is fusion, and I should like to consider loss of fusion after head injury at some length, since it has rarely appeared in the literature. Doden and Bunge (1965) described a series of patients with diminished fusion range, without total loss of fusion; there was eventual normalization of fusion in the majority after orthoptic treatment. Wade (1965) described four adult patients and two children with loss of fusion after head injuries and did not mention any recovery of function; she considered the loss of fusion to be related to intellectual impairment. Hart (1969), from my unit, described six patients with complete loss of fusion with no recovery, five with loss of fusion but full recovery, and four with reduced fusion range with limited stereopsis; he did not find any correlation with intellectual impairment. 
Reduced fusion range and stereopsis is difficult to assess, particularly when legal proceedings and compensation may be factors, so I will concentrate on patients with complete fusion loss.

Case I, a man aged 46 years, was seen 2 weeks after concussion from a motor-cycle accident which had produced anosmia; no fracture had been demonstrated. He had diplopia with a small variable left divergent squint and a very slight vertical deviation: no fusion was present. After 3 days he was able to fuse images to a sufficient degree to convince the orthoptists that more than superimposition was present; another week later fusion was well demonstrated and he had only a slight latent divergence. He finally showed some atrophy of the left optic disc with slight general contraction of the visual field, the visual acuity being 6/9 in the right eye and 6/12 in the left.

This patient demonstrated rapid recovery of fusion after a relatively minor head injury. The next two patients took longer to recover.

Case 2 (Hart's Case 6), a man aged 19 years, was unconscious for 10 days after a road traffic accident, and had a left temporal lobe haematoma. 7 months after the accident there was little if any anomaly of ocular movement apart from slight nystagmus to the left, but no fusion or steropsis was present. 2 months later he had definite fusion but no response to the Wirt stereo-test; 6 months later he had fusion with good stereopsis (6 circles). There was some pallor of the right optic disc with a superonasal quadrantanopia.

Case 3 (Hart's Case 4), a man aged 26 years, suffered a comminuted fracture of the right frontal sinsu in a road traffic accident; the fracture was repaired, but he had right partial IIIrd nerve palsy, and optic atrophy with field loss involving almost all the upper and most of the lower temporal field. 18 months after the accident he had a marked divergent squint with no demonstrable fusion. Right lateral rectus recession and medial rectus resection was carried out, but he continued to have a small divergent squint, with no demonstrable fusion. 8 months after the operation (over 2 years after the accident), he began to show evidence of fusion; temporary prisms were ordered with which he achieved good fusion and stereopsis. In view of the residual divergence and increasing diplopia, a further operation, this time on the left eye, was performed 5 years after the accident, with an excellent final result.

The next patients had much more obvious vertical deviations and have not recovered from the loss of fusion in the period of observation.

Case 4, a man aged 25 years, received multiple injuries in a road traffic accident and was unconscious for 3 or 4 day.. There was full ocular movement but a divergent squint with hypertropia, a form of skew deviation (Fig. I, overleaf). The vision was normal in each eye, but he showed no evidence of fusion and had constant diplopia. 2 years after the accident he had a horizontal muscle operation for cosmetic reasons which greatly reduced the divergence but still left some hypertropia; he still has diplopia and no evidence of fusion 2 months later.

Case 5 (Hart's Case I), a man aged 21 years, was unconscious for 2 to 3 days after a road traffic accident. He had a convergent squint from right VIth nerve palsy, with a vertical deviation which may have been due to partial IIIrd nerve palsy (Fig. 2, overleaf), but may again have been a form of skew deviation. He also had right VIIth nerve palsy leading to poor lid closure, and a sluggish right pupil. He had see-saw vertical nystagmus, mainly seen as intermittent vertical flicks of the right eye. He had no range of fusion and no stereopsis. This persisted for the 12 months he remained under observation. The visual acuity was $6 / 12$ in the right eye and $6 / 6$ in the left, with normal visual fields.

It is of course possible that the fusion in these two patients may improve over a longer period as in the previous patients, but in one further patient who had an accident at the age of 13 years, the loss of fusion has been known to persist for 6 years. 

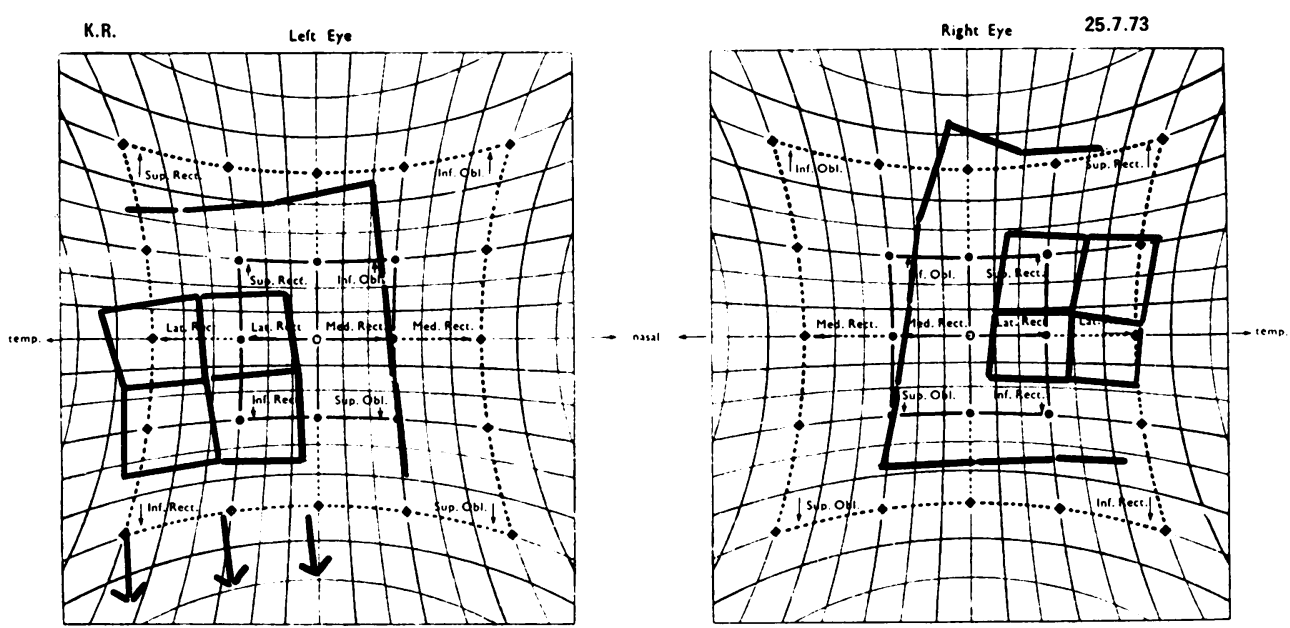

FI G. I Case 4. Skew deviation with loss of fusion
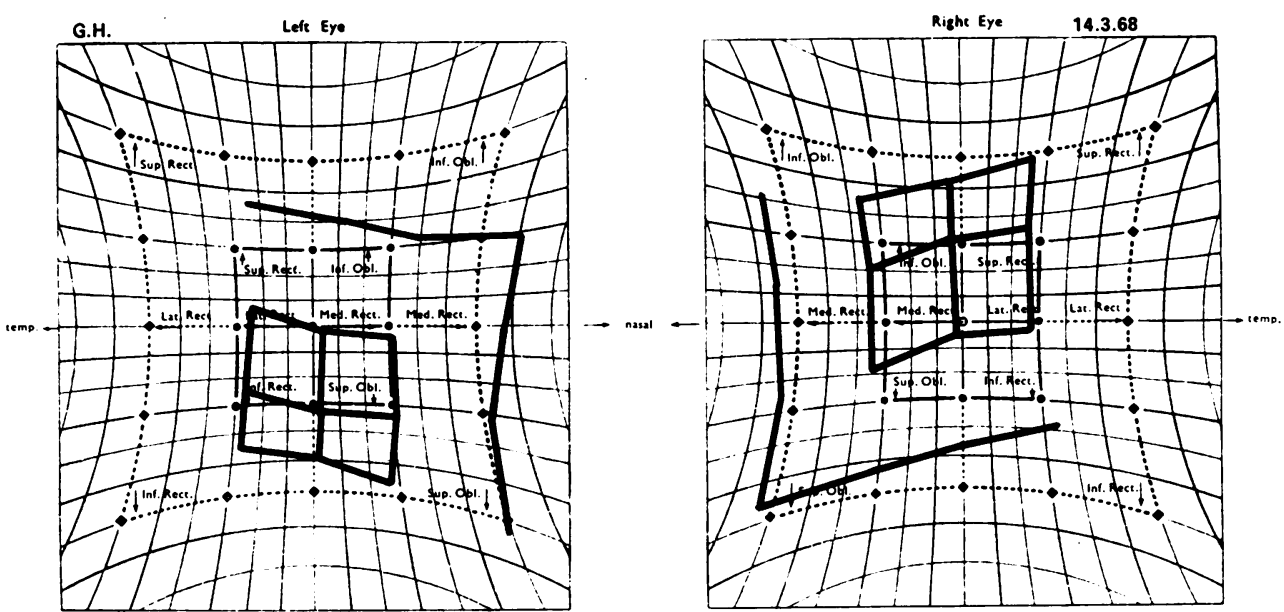

FI G. 2 Case 5. Skew deviation or possible partial IIIrd nerve palsy

I have not recorded convergence and accommodation separately in these patients since these tests are very subjective, variable, and difficult to evaluate. Certainly convergence is often affected in patients with complete loss of fusion, but in Case 2, it appeared surprisingly good at a stage when fusion was still lost, and it may be that recovery of convergence precedes recovery of recordable fusion. Fusion loss is certainly not entirely related to accommodative loss, though unilateral accommodative weakness may make assessment difficult.

Field loss was present in some but not all these patients. Gross field loss can certainly interfere with fusion; this is best seen in patients with bitemporal hemianopia in whom there is only a small overlap of the fields (Kirkham, 1972). In Case 3 the considerable field loss did not prevent the recovery of fusion, and field loss is certainly not the major factor in these patients.

Nystagmus may also make the assessment of fusion difficult; it may have been a factor in Case 5, but was not significant in other patients. 
Gross torsion can also interfere with assessment:

Case 6 (Hart's Case 2), a man aged 21 years, was involved in a mining accident; there was fracture of the frontal bones, and a prolapse of the frontal lobe was evacuated. He had a left divergent squint with gross hypotropia and left intorsion (Fig. 3); he appeared to have loss of fusion, but the difficulty of examination may have been contributory to this.

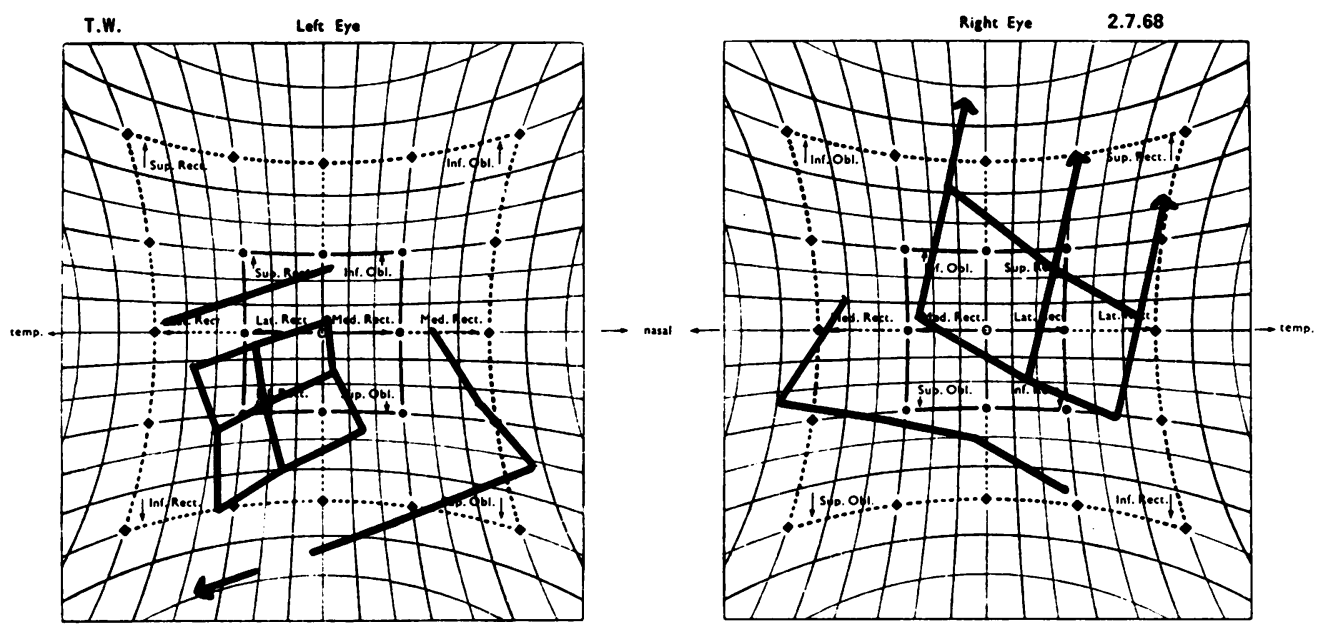

FI G. 3 Case 6. Divergent squint; patient also had gross torsion

This is the type of patient in whom loss of fusion may not always be considered as a possibility, the difficulties in demonstrating fusion in the early stages being ascribed to the torsion and gross deviation, and in the later stages to suppression secondary to the squint. Even in patients with much more typical bilateral superior oblique weakness, some of the initial difficulties may be due to traumatic loss or diminution of fusion rather than directly to torsion.

This brings me to some other factors in the assessment of patients with head injuries. Troublesome torsion is associated with bilateral rather than unilateral superior oblique paresis, but the bilateral nature of the lesion may be missed if the ocular movements are assessed only within a narrow range of movement. In particular, the inner field of the Hess chart may be misleading; Fig. 4 shows a typical bilateral weakness when the patient in question was first seen, but 3 months later its bilateral nature could be missed if only the inner field was considered.

A variety of diagnostic problems may arise in patients who had, or can be presumed to have had, a squint before the head injury. These problems may be relatively easily solved if the previous history is known:

Case 7, a man aged 27 years, had a severe closed-head injury leading to diplopia. He had slight esophoria for distance breaking down to a manifest esotropia, associated with slight limitation of abduction of each eye, but for near he was exophoric, breaking down to a small divergent squint. He had had a convergent squint as a child, which was treated by occlusion only. It appeared likely that he had a long-standing small-angle esotropia, decompensated by the head injury. Bilateral rectus resection removed his diplopia and left him with good stereopsis and right microtropia.

The following case is much more difficult to explain:

Case 8, a man aged 35 years, complained of diplopia after a blow on the head 2 days previously; 
he had not been unconscious. He had a long-standing squint for which an operation had been carried out at the age of I I years, leaving a moderate right convergent squint with hypotropia. There was no obvious added oculo-motor anomaly that could have been due to the head injury; the diplopia persisted with any prism, no area of suppression or evidence of fusion being found. It appeared as if he had lost the power to suppress.

Such loss of suppression is difficult to understand, especially after such a slight injury. Minor trauma may, however, like so many other factors, be the precipitating cause for a squint in children without producing any direct brain damage.

I come finally to a group of patients in whom the head injury was apparently related to a squint but in whom the time relationships were peculiar.

Case 9, a woman aged 44 years, sustained brain and spinal injury with hemiplegia. She was seen 7 months later with a vague history of diplopia in all extremes of gaze, with a variable convergent deviation; the diplopia became worse and she showed a definite limitation of the left lateral rectus muscle. She was fully investigated by a neurosurgeon with no positive findings, and it was assumed that the diplopia was an inexplicably delayed result of the head injury. She was treated with prisms and extraocular muscle surgery. 5 years later she was found to have evidence of disseminated sclerosis.

Case Io, a woman aged 26 years, attended with diplopia after having a road traffic accident 9 months previously. The diplopia was apparent only on extreme dextroversion; she also had convergence weakness for which she was given orthoptic treatment. The diplopia increased in severity, with limitation of left abduction. She was found to have disseminated sclerosis 2 years after the accident.

These two cases suggest the possibility that head trauma may precipitate the onset of the ocular signs of disseminated sclerosis.

Gase II, a man aged 4I, was unconscious for I week and suffered from hemiplegia after a road traffic accident. He had some diplopia from slight bilateral superior oblique weakness which, together with his hemiplegia, made walking difficult. His convergence was good. 14 months after the accident he was found to have no convergence; fusion was present on lateroversions; but 4 months later fusion could not be demonstrated. The fusion began to improve after 5 months (almost 2 years after the accident) and was quite strong I month later. Temporary prisms were prescribed and he eventually achieved good fusion and stereopsis without them.

It is tempting to postulate that this patient had some unrelated disease to account for the course of these anomalies. Eyster, Hoyt, and Wilson (1972) have described three patients in whom minor head trauma, insufficient to cause a fracture or loss of consciousness, was associated with IIIrd nerve palsy; these patients had a parasellar or clival tumour, and Eyster postulates that the IIIrd nerve was stretched over the tumour or partially fixed or encased, so making it more vulnerable to minor stress. In any case of head injury in which the degree of trauma seems inadequate to account for the findings, or in which the time relationships are unusual, the possibility of additional pathological processes must be considered.

In summary, I have tried in this paper to draw attention to some unusual factors in the assessment of head injuries, and to indicate a number of sources of confusion in the diagnosis and management of these often difficult and tragic patients.

I must thank Mr. G. A. L. Palmer and Mr. N. L. McNeil for permission to describe their patients, and particularly Miss Mein and the staff of the Orthoptic Department of the United Sheffield Hospitals without whom this paper could not have been written. 

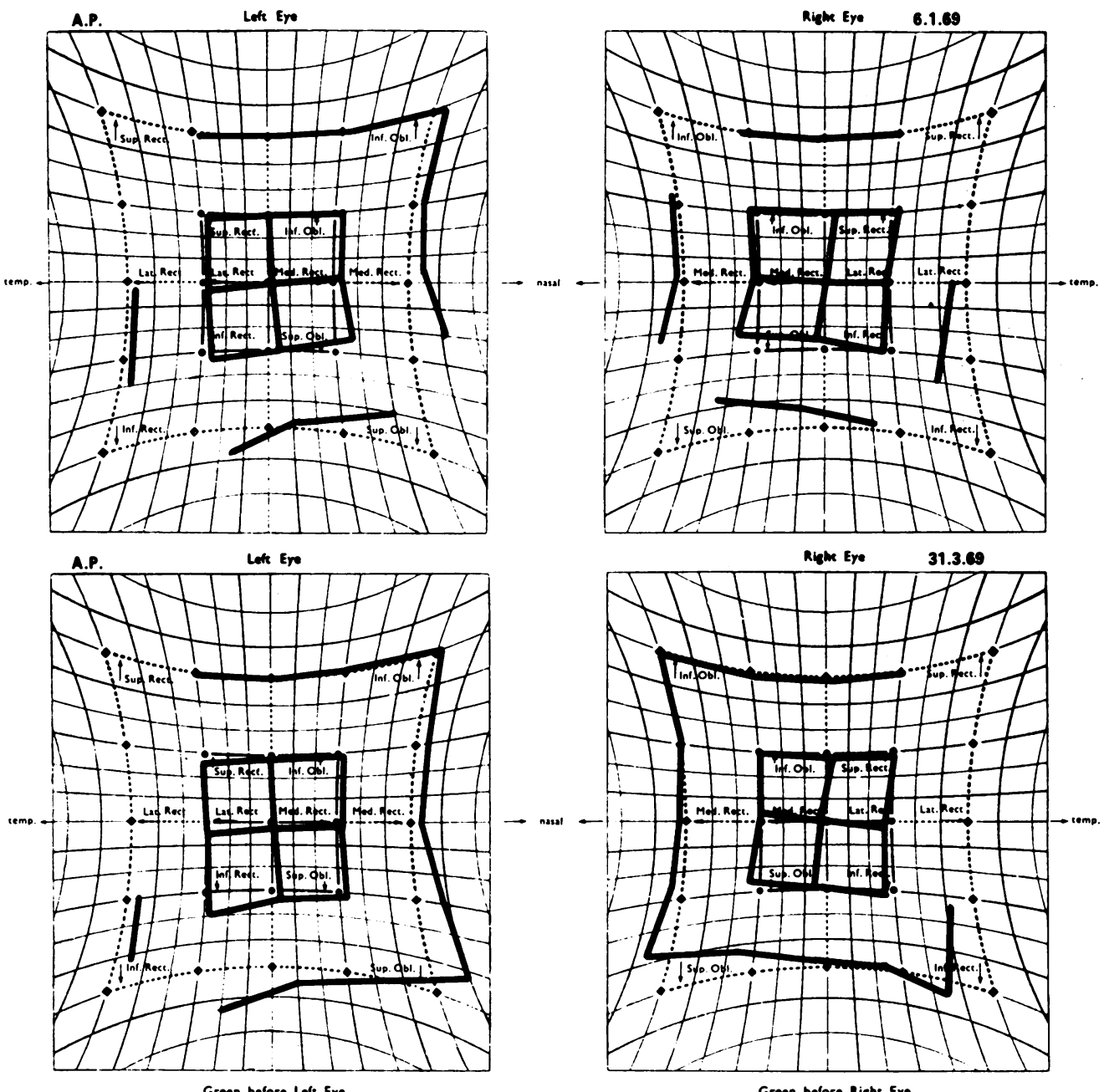

FI G. 4 Case II in Fanuary and March, 1969. Bilateral superior oblique weakness, eventually showing bilateral weakness only in the outer field 